\title{
Rescue extracorporeal membrane oxygenation for massive anterior mediastinal masses
}

\author{
Lowell Leow $^{1}\left[\right.$ ] Hari Kumar Sampath ${ }^{1} \cdot$ Keith J Yong $^{2} \cdot$ Theo Kofidis $^{1,2} \cdot$ John Kit Chung Tam $^{1,2} \cdot$ Graeme MacLaren $^{1}$. \\ Lynette $\mathrm{Teo}^{3} \cdot$ Harish Mithiran ${ }^{1} \cdot$ Kollengode Ramanathan ${ }^{1}$
}

Received: 10 June 2020 / Accepted: 25 March 2021 / Published online: 3 April 2021

(c) The Japanese Society for Artificial Organs 2021

\begin{abstract}
The management of massive anterior mediastinal masses (AMM) is challenging. With the burgeoning role of extracorporeal membrane oxygenation support (ECMO) beyond the confines of salvage therapy, more trained clinicians are adopting it as a bridge for high-risk procedures or situations where temporary respiratory or cardiac support is required. We report our experience with using ECMO in the management of massive AMM in this case series of three patients sharing their clinical details and the lessons learned from them.
\end{abstract}

Keywords ECMO $\cdot$ Anterior mediastinal mass $\cdot$ Mediastinal mass

\section{Introduction}

The management of massive anterior mediastinal masses (AMM) poses both a diagnostic and therapeutic challenge. The symptoms and signs vary from patients being asymptomatic to presenting in extremis secondary to compression on mediastinal structures. The superior vena cava (SVC), pulmonary arteries (PA), and trachea are compressed in that order because of their inherent wall tension. Due to the slow-growing nature of AMMs, patients experience symptoms insidiously. Radiological diagnosis of AMM is easily made with computed tomography (CT) scans but the histological diagnosis that further guides treatment entails patients undergoing a biopsy. The anaesthetic considerations

Lowell Leow

lowell_leow@nuhs.edu.sg; lowell.leow@gmail.com

Kollengode Ramanathan

ram_ramanathan@nuhs.edu.sg

1 Department of Cardiac, Thoracic and Vascular Surgery, National University Heart Centre Singapore, 5 Lower Kent Ridge Road, Singapore 119074, Singapore

2 Department of Surgery, Yong Loo Lin School of Medicine, National University of Singapore, Singapore, Singapore

3 Department of Diagnostic Imaging, National University Hospital, Singapore, Singapore in patients with AMM are complex and have been widely discussed [1]. Extracorporeal membrane oxygenation (ECMO) has been described in reports as a successful means of bridging patients with AMMs causing extrinsic airway compression during induction chemotherapy or surgical treatment [2-15]. We present a series of patients who underwent Veno-arterial (VA) ECMO for management of their AMM and review literature on the use of this modality. We share our threshold for initiating ECMO in patients with massive AMM and encourage early adoption of ECMO to improve survival.

\section{Case series}

The study has obtained local DSRB approval (2018/01257). Waiver of consent was given.

\section{Case 1}

A 40-year-old lady with a large left goitre with retrosternal extension presented with compressive symptoms of dysphagia, shortness of breath, and stridor. She was unable to lie flat and slept prone. Due to her symptoms, surgical excision was offered. CT thorax showed a $11.6 \times 11.0 \mathrm{~cm}$ left thyroid lobe nodule with mass effect displacing the left sternocleidomastoid muscle, internal jugular vein, common 


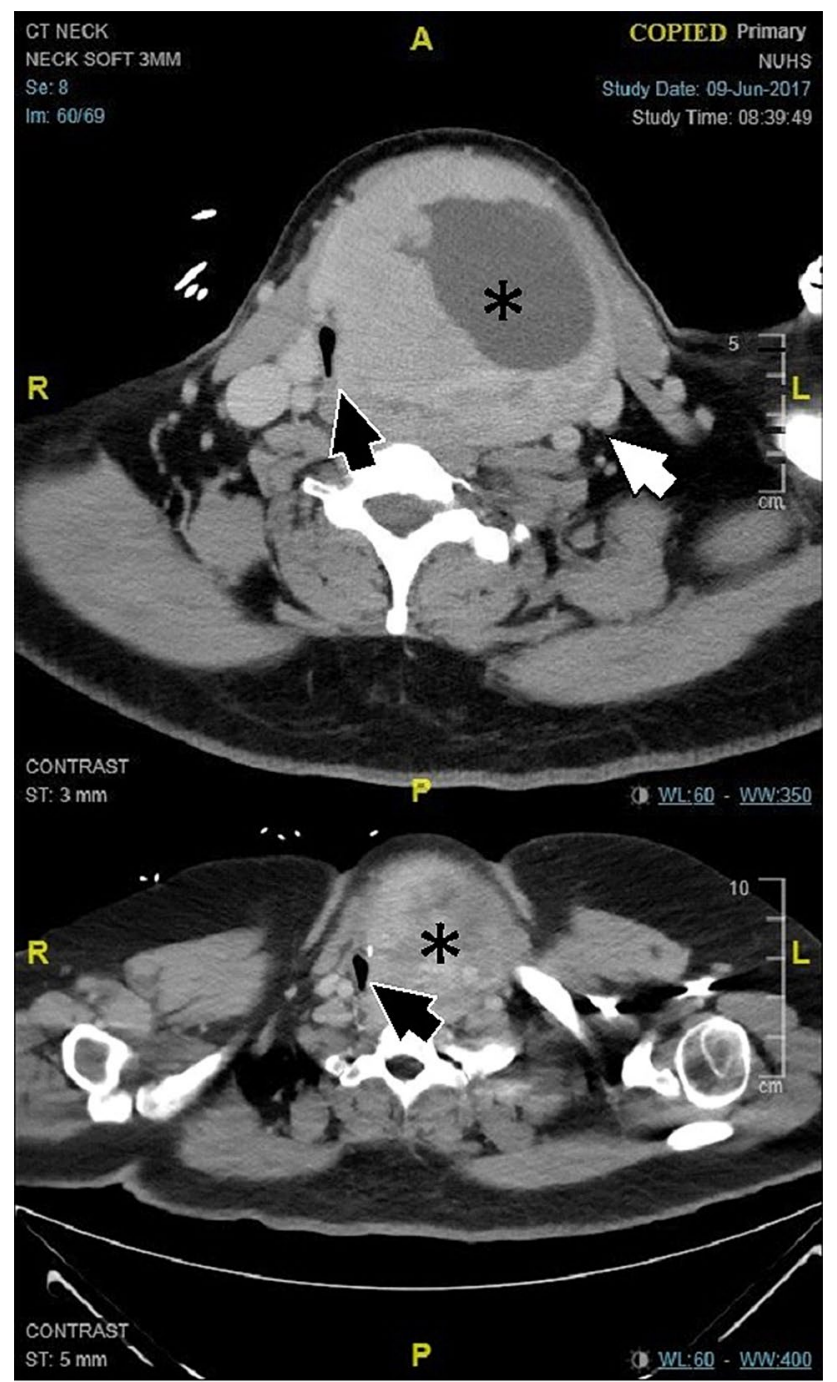

Fig. 1 CT Thorax showing large goitre (*) with displacement of carotid vessels (white arrow) and compression of trachea (black arrow)

carotid artery and severe narrowing of the trachea (Fig. 1). In view of the airway risk, ECMO support was activated for the left hemithyroidectomy. A multidisciplinary discussion was held where it was discussed that in the event of failed intubation, veno-venous (VV) ECMO would be initiated. The patient could not be intubated despite attempts at awake fibreoptic intubation. Thus VV ECMO support was started in lieu of ventilation. Bilateral femoral veins were cannulated by open cut down, in view of the patient's large body habitus (body mass index 34.6), under local anaesthesia. $3.5 \mathrm{~L} / \mathrm{min}$ of flows were achieved with the drainage cannula in the inferior vena cava and the return cannula in the right atrium. The cannulas were positioned appropriately so as to exclude recirculation. However, this was inadequate to maintain oxygenation and the cannulation strategy was

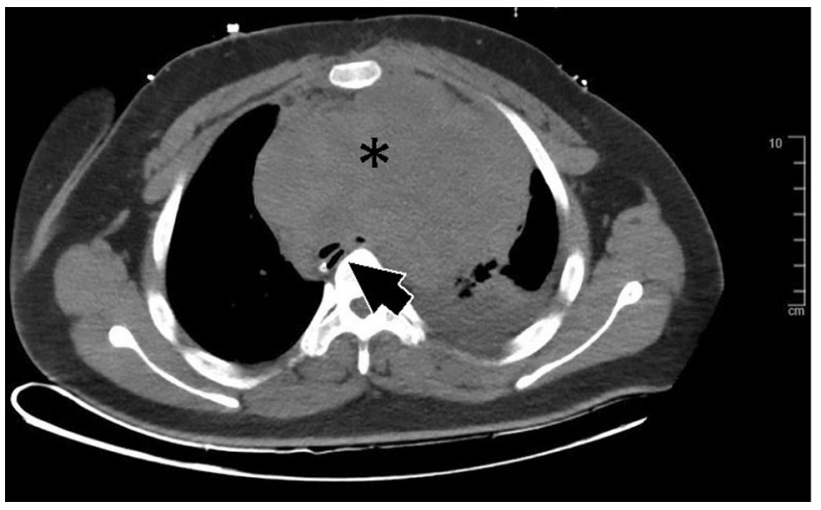

Fig. 2 CT Thorax showing AMM (*) compressing trachea (black arrow) and obliterating the SVC and brachiceophalic vein

changed to femoral VV-A ECMO. This permitted higher flows of $5.5 \mathrm{~L} / \mathrm{min}$ but the patient had differential hypoxemia with right upper limb saturation remaining at $60 \%$. A semi emergent tracheostomy was performed which enabled better ventilation and improved the saturations to above $90 \%$. The left thyroidectomy was then performed under ECMO support and the patient was weaned off ECMO post-operatively. Post-operatively patient recovered and her tracheostomy decannulated prior to discharge on day 24 .

\section{Case 2}

A 23-year-old gentleman presented with worsening shortness of breath, stridor, and hoarseness of voice. He was diagnosed 6 months prior with germ cell tumour of the mediastinum but defaulted follow-up. CT scan then showed a $14.0 \times 10.0 \mathrm{~cm}$ AMM with SVC obstruction, PA and airway compromise. A repeat $\mathrm{CT}$ after 6 months showed that the mass had increased to $16.0 \times 13.0 \mathrm{~cm}$ with severe compression of great vessels, airway, and left phrenic nerve causing diaphragmatic palsy (Fig. 2). Due to his impending airway compromise, his right femoral artery and vein were percutaneously cannulated with a 7Fr sheath in preparation for ECMO. He underwent awake fibreoptic intubation with a size 8.5 armoured cuff endotracheal tube (ETT) and bronchoscopy in the operating theatre. Distal to the ETT, the carina was compressed $80 \%$ in diameter, the left main bronchus compressed $70 \%$ and right main bronchus compressed $90 \%$. The distal bronchi were patent. The patient was transferred to the intensive care unit (ICU) after intubation but remained unstable with difficulties in ventilation. The ETT was adjusted under bronchoscopic guidance beyond the collapsed part of the trachea but the patient deteriorated and had $\mathrm{a}<1$ min episode of pulseless electrical activity arrest. He was resuscitated but required inotropic support (Noradrenaline $0.1 \mathrm{mcg} / \mathrm{kg} / \mathrm{min}$ ) and had poor gas exchange. In view of 
the haemodynamic instability and possibility of worsening tumour size after initiation of chemotherapy, decision was made to stabilise the patient on VA ECMO. VA ECMO was initiated via the cannulated femoral vessels. Chemotherapy was started and serial CTs showed a decrease in the size of the AMM. He was decannulated on day 8. However, he failed extubation due to tracheal and bronchomalacia for which he underwent both tracheal and bronchial stent insertion. He was eventually extubated on day 39 . As a result of immunosuppression, he sustained an infected pseudoaneurysm of his femoral artery which required repair with a pericardial patch and sartorius muscle flap. The patient was discharged from the hospital on day 58 .

\section{Case 3}

A 29-year-old gentleman presented with progressive facial swelling over a month associated with shortness of breath, productive cough, orthopnoea, and unintentional weight loss. CT Thorax showed a $14.1 \times 11.7 \mathrm{~cm}$ AMM causing SVC obstruction, compression of bilateral brachiocephalic trunks, thrombosis of the right internal jugular vein, and distortion of the main and bilateral PAs (Fig. 3). He underwent bilateral anterior mediastinotomy and biopsy of AMM with ECMO standby support. The left femoral artery and vein were percutaneously cannulated with 7Fr sheaths. During the procedure, the patient desaturated was intubated and initiated on femoral VA ECMO support. The surgical biopsy was completed and he was shifted to ICU for further management. Histology revealed primary mediastinal B-cell lymphoma. He was initiated on chemotherapy and intravenous steroids with prophylaxis for tumour lysis syndrome. The tumour size did not change despite two cycles until he received a fraction of radiotherapy. He was decannulated after 12 days of VA ECMO. Serial CTs showed improvement in mass effect on the trachea and PAs. He had difficulty weaning off the ventilator requiring multiple bronchoscopies to evacuate bronchial clots and secretions to open up the distal bronchi. His left mediastinotomy wound became infected with Elizabethkingia requiring VAC dressing and Levofloxacin. The patient eventually recovered and was discharged home on day 140 .

\section{Discussion}

We share our experience of three patients with AMM who successfully had ECMO for hemodynamic support as part of their diagnostic and therapeutic management. The mortality of two of our patients prompted us to recalibrate our approach towards earlier ECMO team activation and create a workflow for high-risk patients with AMM (Table 1). We observed that delayed diagnosis of massive AMM, late

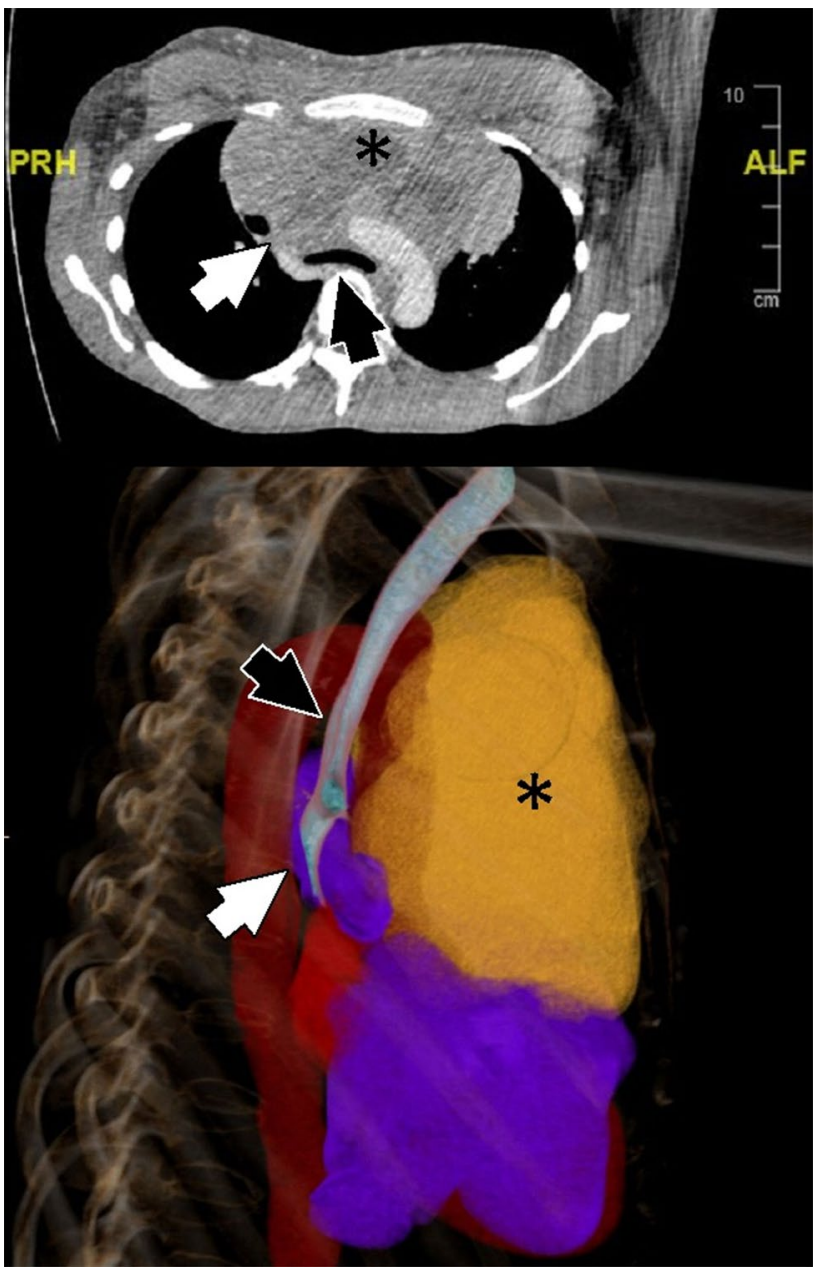

Fig. 3 CT Thorax and 3D reconstruction showing AMM (*) causing tracheal compression (black arrow) and SVC compression (white arrow)

referrals for ECMO, and lack of preparedness contributed to mortality. Our first mortality (MABS) was in a, young patient who collapsed in the haematology procedure room when he was sedated and positioned for a bone marrow aspirate in the right lateral position. When patients with massive AMM are deeply sedated or paralyzed, the weight of the AMM causes acute airway or great vessel occlusion precipitating cardiopulmonary compromise. Cardiopulmonary resuscitation was started and the decision for ECMO activation was delayed. By the time ECMO was initiated, it may have been too late and patient's heart and brain had infarcted. Our second patient (MSBR), who died, had an open biopsy in the operating theatre with anaesthesia support. Unfortunately, massive tumour bleeding was encountered during the procedure which rapidly expanded the tumour and exacerbated the compressive symptoms. While the call for ECMO initiation was made expediently, access lines were not yet placed and the team had to be called in during out-of-office hours. This 
Table 1 Summary of cases with massive AMM requiring ECMO

\begin{tabular}{|c|c|c|c|c|c|}
\hline Patient & MABS & MSBR & CSL & $\mathrm{BH}$ & NHL \\
\hline Age & 19 & 23 & 40 & 23 & 29 \\
\hline Gender & Male & Male & Female & Male & Male \\
\hline Weight (kg) & 60 & 101 & 100 & 100 & 60 \\
\hline Height $(\mathrm{cm})$ & 170 & 174 & 170 & 180 & 164 \\
\hline BSA & 1.70 & 2.15 & 2.10 & 2.20 & 1.65 \\
\hline Size of mass $(\mathrm{cm})$ & $12.0 \times 8.8$ & $13.2 \times 10.6$ & $11.6 \times 11.0$ & $16.0 \times 13.0$ & $14.1 \times 11.7$ \\
\hline $\begin{array}{l}\text { Compression of } \\
\text { airway }\end{array}$ & Yes & Yes & Yes & Yes & Yes \\
\hline Compression of SVC & No & Yes & No & Yes & Yes \\
\hline $\begin{array}{l}\text { Compression of great } \\
\text { arteries }\end{array}$ & Yes & No & No & Yes & Yes \\
\hline $\begin{array}{c}\text { Compression of } \\
\text { phrenic nerve }\end{array}$ & No & No & No & Yes & No \\
\hline Bleeding & Yes & No & No & No & No \\
\hline Etiology of mass & $\begin{array}{l}\text { Acute lymphoblastic } \\
\text { leukaemia }\end{array}$ & $\begin{array}{l}\text { Diffuse large B cell } \\
\text { lymphoma }\end{array}$ & Retrosternal goitre & Germ cell tumour & $\begin{array}{l}\text { Primary mediastinal } \\
\text { B-cell lymphoma }\end{array}$ \\
\hline Surgery & Bone marrow aspirate & $\begin{array}{l}\text { Chamberlain under } \\
\text { LA convert emer- } \\
\text { gency right hemi- } \\
\text { clamshell }\end{array}$ & $\begin{array}{l}\text { Left hemithyroidec- } \\
\text { tomy }\end{array}$ & $\begin{array}{l}\text { Awake fibreoptic } \\
\text { intubation }\end{array}$ & $\begin{array}{l}\text { Bilateral chamberlain } \\
\text { under LA }\end{array}$ \\
\hline ECMO type & VA ECMO & VA ECMO & VV to VVA ECMO & VA ECMO & VA ECMO \\
\hline Cannulation strategy & $\begin{array}{l}\text { Percutaneous RCFV } \\
\text { then cutdown RCFA }\end{array}$ & $\begin{array}{l}\text { Right groin cutdown } \\
\text { for RCFA + RCFV }\end{array}$ & $\begin{array}{l}\text { Bilateral groin } \\
\text { cutdown, bilateral } \\
\text { femoral veins then } \\
\text { RCFA }\end{array}$ & $\begin{array}{l}\text { Right groin cutdown } \\
\text { for RCFA + RCFV }\end{array}$ & $\begin{array}{l}\text { Right groin cutdown } \\
\text { for RCFA + RCFV }\end{array}$ \\
\hline $\begin{array}{l}\text { Duration of ECMO } \\
\text { run (days) }\end{array}$ & 1 & 1 & 1 & 8 & 12 \\
\hline Weaned off ECMO & No & No & Yes & Yes & Yes \\
\hline Inpatient mortality & Yes & Yes & No & No & No \\
\hline On ECMO mortality & Yes & Yes & No & No & No \\
\hline ECMO site infection & No & No & No & Yes & Yes \\
\hline
\end{tabular}

delay contributed to the patient's eventual demise. Through multiple root cause analyses and multidisciplinary discussions, we formulated a protocol (Fig. 4) which involved early consultation and involvement by oncologists, intensivists, radiologists, anaesthetists, and cardiothoracic surgeons once a patient is diagnosed with an AMM. Patients identified as high-risk would undergo surgical biopsy in the operating theatre with anaesthesia and standby ECMO support. Femoral access sheaths are inserted under local anaesthesia prior to biopsy to expedite ECMO initiation when necessary. A primed ECMO circuit is set up in the theatre as the case is underway. The patient undergoes awake fibreoptic intubation, if needed, to secure the airway. Should intubation fail or patient develop peri-arrest symptoms, ECMO is immediately instituted with cannulation under local anaesthesia. After the biopsy, the patient is monitored in a high dependency unit. If no cannulation occurs, the groin lines are kept postoperatively for $24 \mathrm{~h}$ to ensure the patient tolerates induction therapy. Thirteen patients were referred after the protocol was devised of which the three patients in this series went on ECMO.

There is an increasing role of ECMO beyond the confines of salvage therapy as a bridge for temporary cardiopulmonary stabilization. This can be applied to patients deemed high risk for cardiac arrest when undergoing procedures. For patients with AMM, these include anterior mediastinotomy or radiological guided core biopsies. ECMO use has been well described for patients with lymphoma requiring invasive biopsy for histology [2-6]. More recently, ECMO has been used intraoperatively for cardiopulmonary support of patients with anticipated difficulties of securing airway or ventilation [7]. The use of ECMO in a subtotal thyroidectomy with distorted airway as a means for oxygenation has been reported [8]. Others have also reported the use of ECMO for the duration of surgery when resecting mediastinal and bronchoscopic masses [9-11]. Placing the patient on ECMO eliminates the need for constant airway ventilation during surgery allowing for better oncological resection. 


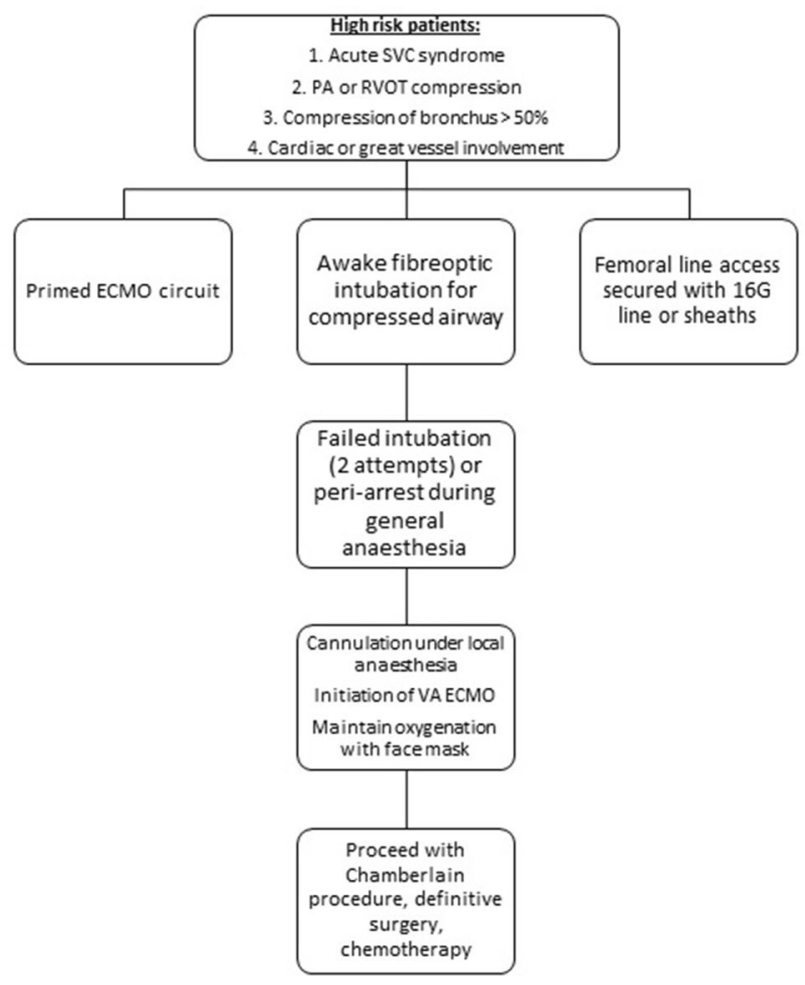

Fig. 4 Simplified mediastinal mass protocol and indications for ECMO

Beyond these procedures, ECMO has also been used in a supportive role for patients with AMM compromising cardiopulmonary function. In a reported series of lymphoma patients two of them required ECMO for high-risk mediastinal masses [12]. These patients had severe compression of their PA and SVC which needed stenting on ECMO. Other authors have also reported similar cases of patients needing ECMO for mediastinal masses [13-15]. These cases are summarized in Table 2.

The choice of ECMO mode in such scenarios can be challenging. Several case reports highlight patients with mediastinal masses who were managed either on VV or VA ECMO [8, 9, 14]. VA ECMO would be prudent in patients with extrinsic compression on the airways and vasculature as well as during an emergent peri-arrest setting [8, 14]. Tracheal or bronchial tumours with fixed airway obstruction may be managed on VV ECMO [9]. If in doubt, we recommend insertion of VA ECMO first with a view for an additional venous cannula if required [16]. As seen in case 1, we attempted VV initially but were unable to sustain oxygenation with just venous cannulas. Hence we changed strategy to VVA to improve drainage and thereby provide better flows and improve oxygenation. Patients with AMM have altered neck anatomy or superior venacaval syndrome making additional venous access difficult. In a patient with good cardiac output, this can lead to differential hypoxia, however, it might afford sufficient time for a definitive airway or a tracheostomy to be performed. We encourage open multidisciplinary discussions prior to embarking on such endeavours and to establish clear thresholds not only for instituting but also withdrawing ECMO support [16]. Differential hypoxia should be routinely looked for in all patients with VA ECMO especially if cardiac function remains preserved.

In our experience, the major challenge encountered was that of cannula site infection in these immunocompromised patients. We practice a groin cut down for VA ECMO for better exposure during cannulation, decannulation and repair but this poses a higher infection risk. We mitigate this by cannulating in an aseptic manner, vigilant wound care and treating any potential infection aggressively.

Both percutaneous and cut-down cannulations are viable options depending on a centre's operators' preference and familiarity. Both are shown to have similar outcomes with less infection for percutaneous closure but more vascular complications on decannulation [17]. Percutaneous insertion requires the use of ultrasound image-guided insertion which may be less invasive but requires competency with ultrasound usage. Cut down requires surgical instruments and techniques that non-surgically trained personnel may not be familiar with. Traditionally, both would require femoral artery repair upon decannulation of arterial cannulas. With the advent of perclose devices, percutaneous cannulations may save patients an additional surgery upon decannulation [18]. We recommend that ECMO cannulators adopt the strategy that they are most competent with and prepared for with the necessary equipment available.

\section{Conclusion}

Our case series presents adult patients with massive AMM that underwent VA ECMO. We advocate for the use of ECMO in AMM both as a supportive and rescue measure. We believe that as ECMO is further refined and its deleterious effects mitigated, the use of ECMO can be expanded to incorporate wider indications and adopted in a more preventive and elective setting. Our protocol seeks to identify high-risk patients from the outset based on both clinical and radiological criteria. It specifies clearly the multiprong approach that ECMO teams can take in ensuring the safety of patients with AMM.

Acknowledgements We acknowledge the perfusion team at the National University Heart Centre Singapore for their support of our ECMO programme and all other persons involved in the formulation of the workflow.

Author contributions All authors contributed to the study conception and design. Material preparation, data collection, and analysis were 
Journal of Artificial Organs (2021) 24:450-457

455

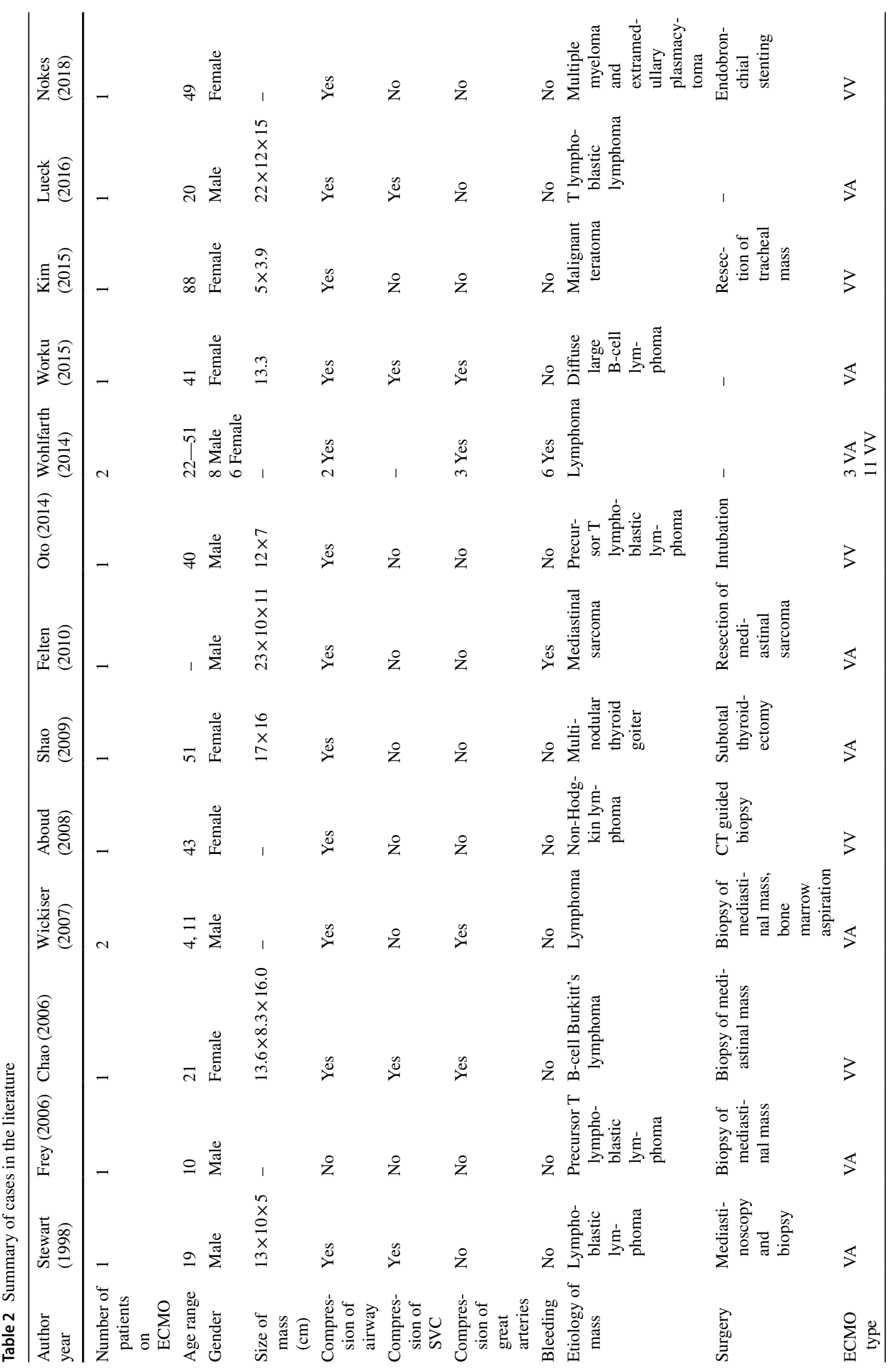

Springer 
456

Journal of Artificial Organs (2021) 24:450-457

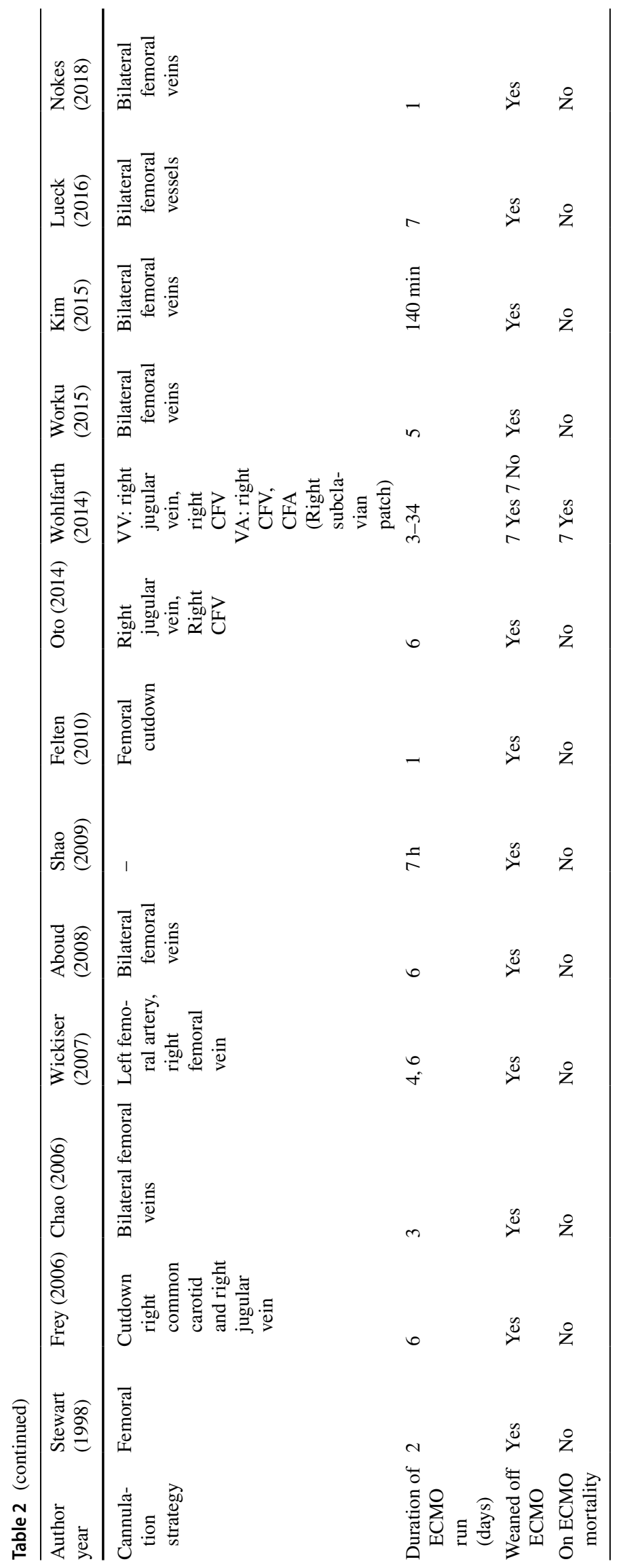

Springer 
performed by LL, HKS, KYJ, LT, HM, and KR. The first draft of the manuscript was written by LL and all authors commented on previous versions of the manuscript. All authors read and approved the final manuscript.

Funding None.

\section{References}

1. Gothard JW. Anesthetic considerations for patients with anterior mediastinal masses. Anesthesiol Clin. 2008;26:305-14.

2. Stewart AS, Smythe WR, Aukburg S, Kaiser LR, Fox KR, Bavaria JE. Severe acute extrinsic airway compression by mediastinal tumor successfully managed with extracorporeal membrane oxygenation. ASAIO J. 1998;44:219-21.

3. Frey TK, Chopra A, Lin RJ, et al. A child with anterior mediastinal mass supported with veno-arterial extracorporeal membrane oxygenation. Pediatr Crit Care Med. 2006;7:479-81.

4. Chao VT, Lim DW, Tao M, Thirugnanam A, Koong HN, Lim $\mathrm{CH}$. Tracheobronchial obstruction as a result of mediastinal mass. Asian Cardiovasc Thorac Ann. 2006;14:e17-8.

5. Wickiser JE, Thompson M, Leavey PJ, Quinn CT, Garcia NM, Aquino VM. Extracorporeal membrane oxygenation (ECMO) initiation without intubation in two children with mediastinal malignancy. Pediatr Blood Cancer. 2007;49(5):751-4.

6. Aboud A, Marx G, Sayer H, Gummert JF. Successful treatment of an aggressive non-Hodgkin's lymphoma associated with acute respiratory insufficiency using extracorporeal membrane oxygenation. Interact Cardiovasc Thorac Surg. 2008;7(1):173-4.

7. Foong TW, Ramanathan RK, Chan KK, MacLaren G. Extracorporeal membrane oxygenation during adult non-cardiac surgery and perioperative emergencies: a narrative review. J Cardiothorac Vasc Anesth. 2020. https://doi.org/10.1053/j.jvca.2020.01.025.

8. Shao Y, Shen M, Ding Z, Liang Y, Zhang S. Extracorporeal membrane oxygenation-assisted resection of goiter causing severe extrinsic airway compression. Ann Thorac Surg. 2009;88:659-61.

9. Nokes BT, Vaszar L, Jahanyar J, Swanson KL. VV-ECMOassisted high-risk endobronchial stenting as rescue for asphyxiating mediastinal mass. J Bronchol Interv Pulmonol. 2018;25:144-7.
10. Kim JJ, Moon SW, Kim YH, Choi SY, Jeong SC. Flexible bronchoscopic excision of a tracheal mass under extracorporeal membrane oxygenation. J Thorac Dis. 2015;7:E54.

11. Felten ML, Michel-Cherqui M, Puyo P, Fischler M. Extracorporeal membrane oxygenation use for mediastinal tumor resection. Ann Thorac Surg. 2010;89:1012.

12. Wohlfarth P, Ullrich R, Staudinger T, et al. Extracorporeal membrane oxygenation in adult patients with hematologic malignancies and severe acute respiratory failure. Crit Care. 2014;18:R20.

13. Lueck C, Kuehn C, Hoeper MM, Ganser A, Eder M, Beutel G. Successful use of extracorporeal membrane oxygenation during induction chemotherapy in a patient with mediastinal tumor mass of a T lymphoblastic lymphoma. Ann Hematol. 2016;95:1719-21.

14. Worku B, DeBois W, Sobol I, Gulkarov I, Horn EM, Salemi A. Extracorporeal membrane oxygenation as a bridge through chemotherapy in B-cell lymphoma. J Extra Corpor Technol. 2015;47:52.

15. Oto M, Inadomi $\mathrm{K}$, Chosa $\mathrm{T}$, Uneda $\mathrm{S}$, Uekihara $\mathrm{S}$, Yoshida $\mathrm{M}$. Successful use of extracorporeal membrane oxygenation for respiratory failure caused by mediastinal precursor $\mathrm{T}$ lymphoblastic lymphoma. Case Rep Med. 2014. https://doi.org/10.1155/2014/ 804917.

16. Ramanathan K, Leow L, Mithiran H. ECMO and adult mediastinal masses. Indian J Thorac Cardiovasc Surg. 2021. https://doi.org/ 10.1007/s12055-020-01077-x.

17. Danial P, Hajage D, Nguyen LS, Mastroianni C, Demondion P, Schmidt M, Bouglé A, Amour J, Leprince P, Combes A, Lebreton G. Percutaneous versus surgical femoro-femoral veno-arterial ECMO: a propensity score matched study. Intensive Care Med. 2018;44(12):2153-61.

18. Hwang JW, Yang JH, Sung K, Song YB, Hahn JY, Choi JH, Gwon HC, Choi SH. Percutaneous removal using Perclose ProGlide closure devices versus surgical removal for weaning after percutaneous cannulation for venoarterial extracorporeal membrane oxygenation. J Vasc Surg. 2016;63(4):998-1003.

Publisher's Note Springer Nature remains neutral with regard to jurisdictional claims in published maps and institutional affiliations. 